\title{
On certain continued fraction expansions of fixed period length
}

\author{
by
}

\section{A. J. VAN DER Poorten (Sydney, N.S.W.) and H. C. Williams (Winnipeg, Man.)}

1. Introduction. It is an interesting problem to detect infinite families of positive integers $D$ for which one can readily describe the fundamental unit of the quadratic number field $\mathbb{Q}(\sqrt{D})$. We will discuss here a class of cases $D=F(X)$ with $F$ a polynomial of even degree and with leading coefficient a square, for which one obtains particularly small units, essentially because the period length is independent of the integer parameter $X$. That of course means a particularly large class number for the field $\mathbb{Q}(\sqrt{D})$.

The context is a result of Schinzel [4], [5], who shows that if $F$ is an integer-valued polynomial, either of odd degree, or of even degree with its leading coefficient not a square, then as the integer $X$ varies one has $\varlimsup \lim (\sqrt{F(X)})=\infty$; here $\operatorname{lp}(\delta)$ denotes the length of the period of the continued fraction expansion of the quadratic irrational $\delta$. On the other hand, in the quadratic case Schinzel shows that $\varlimsup \lim \operatorname{lp}(\sqrt{F(X)})<\infty$ if and only if $F(X)=A^{2} X^{2}+B X+C$ with $A>0$, discriminant $\Delta=B^{2}-4 A^{2} C \neq 0$ and $\Delta \mid 4\left(2 A^{2}, B\right)^{2}$. Well known examples of such $F$ include the Richaud-Degert types: $A^{2} X^{2} \pm A, A^{2} X^{2} \pm 2 A$, and $A^{2} X^{2} \pm 4 A$, which provide periods of length at most 12 . As these Richaud-Degert types have been fully investigated (see, for example, Theorem 3.2.1 of Mollin [1]), we will exclude them from our investigations here.

It will also be convenient to deal only with those $F(X)$ such that $2 \mid A$ and $2 \mid B$. There is no loss of generality in doing so as we can divide the possible values of $X$ into even $(X=2 W)$ or odd $(X=2 W+1)$ integers and

1991 Mathematics Subject Classification: Primary 11A55; Secondary 30 B70.

Key words and phrases: periodic continued fraction, period length.

The work of the first author was supported in part by a grant from the Australian Research Council, whilst the research of the second author was supported by NSERC Canada grant \#A7649. 
write

$$
F(X)=G(W)=A^{\prime 2} W^{2}+B^{\prime} W+C^{\prime},
$$

where, if $X=2 W$,

$$
A^{\prime}=2 A, \quad B^{\prime}=2 B, \quad C^{\prime}=C
$$

or, if $X=2 W+1$,

$$
A^{\prime}=2 A, \quad B^{\prime}=4 A^{2}+2 B, \quad C^{\prime}=A^{2}+B+C .
$$

In either case we get $\Delta^{\prime}=B^{\prime 2}-A^{\prime 2} C=4 \Delta$ and $2\left(2 A^{2}, B\right) \mid\left(2 A^{\prime 2}, B^{\prime}\right)$; hence $\Delta^{\prime} \mid 4\left(2 A^{\prime 2}, B^{\prime}\right)^{2}$ whenever $\Delta \mid 4\left(2 A^{2}, B\right)^{2}$. As we may always assume that $2 \mid B$ it will be convenient in what follows to replace $B$ by $2 B$ in $F(X)$ and rewrite it as

$$
F(X)=A^{2} X^{2}+2 B X+C .
$$

In this case Schinzel's condition becomes $B^{2}-A^{2} C \mid 4\left(A^{2}, B\right)^{2}$.

In [6], Stender determines the fundamental unit of $\mathbb{Q}(\sqrt{D})$ when $D=$ $F(X)$ with $F$ quadratic as above, provided that $D$ is squarefree. In this paper we consider the quadratic case only. We find that for $X>0$, Schinzel's condition, together with $\left(A^{2}, 2 B, C\right)$ squarefree, entails that the "approximation" $A X+B / A$ to $\sqrt{F(X)}$ usually provides the first half of a period of $\sqrt{F(X)}$. Thus, aside from some possibly degenerate cases with $X$ small and a special case we are about to allude to, the period of $\sqrt{F(X)}$ is not just of bounded, but in fact of constant length. However, if $F(X) \equiv 1 \bmod 4$ and both the numerator and denominator, after division by the greatest common divisor of $A$ and $B$, of the approximation $A X+B / A$ are odd, then the expansion of that approximation provides just the first sixth of the period. Indeed, we shall show that, under our conditions, if $C \leq 0$ or $C$ is a perfect square, then $F(X)$ is of Richaud-Degert type; but if $C$ is positive and not a square then the continued fraction expansion of $\sqrt{F(X)}$ can usually be expressed very simply in terms of the continued fraction of $\sqrt{C}$. Furthermore, we show that no matter how large a value of $N$ is selected there are always some $A, B, C$ obeying Schinzel's condition such that $\operatorname{lp}(\sqrt{F(X)})>N$. Furthermore, this value of the period length is independent of $X$ as long as $X$ is large enough to avoid some degenerate cases. For example, we must have $X$ large enough that $F(X)$ cannot be a perfect square; this will certainly be the case if $2\left(A^{2} X+|B|\right)>|\Delta|$. We should mention that some of our results were known to Stern [7], but we will be more general than he and use different techniques.

2. Preliminary observations. To begin our investigation it is necessary to characterise those values of $A, B, C$ such that $B^{2}-A^{2} C \mid 4\left(A^{2}, B\right)^{2}$, and $\left(A^{2}, 2 B, C\right)$ is squarefree. 
Lemma 2.1. Set $S=(A, B)$ and $(B / S)^{2}-(A / S)^{2} C=G^{2} H$, where $H$ is squarefree. If $B^{2}-A^{2} C$ divides $4\left(A^{2}, B\right)^{2}$, then $G H$ divides $2 A, 2 B / S$ and $2 S$, and $G^{2} H$ divides $4\left(A^{2}, 2 B, C\right)$. Therefore if $\left(A^{2}, 2 B, C\right)$ is squarefree, then $G=1,2$.

Proof. Since $G^{2} H \mid 4\left(A^{2} / S, B / S\right)^{2}$ and $(A / S, B / S)=1$, it follows that $G H \mid 2(S, B / S)$. Also, since $G H \mid 2 B / S$ we must have $G^{2} H \mid 4 C$; hence, $G^{2} H \mid 4\left(A^{2}, 2 B, C\right)$.

Theorem 2.2. Assume that $B^{2}-A^{2} C$ divides $4\left(A^{2}, B\right)^{2}$ and $\left(A^{2}, 2 B, C\right)$ is squarefree. Then $F(X)=A^{2} X^{2}+2 B X+C$ is of Richaud-Degert type when $C \leq 0$ or $C$ is a perfect square; that is $F(X)=R^{2}+S$ where $S$ divides $4 R$.

Proof. If $C=0$, then $B \mid 2 A^{2}$ and since $\operatorname{gcd}\left(A^{2}, 2 B\right)$ is squarefree, we must have $B \mid 2 A$ and $F(X)=A^{2} X^{2}+2 B X$ where $2 B X \mid 4 A X$. This is of Richaud-Degert type. If $C<0$, we see from Lemma 2.1 that

$$
H(2 B /(S G H))^{2}+(A / S)^{2} 4|C| /\left(G^{2} H\right)=4 .
$$

We must have $H>0$ and $H(2 B /(S G H))^{2} \leq 3$; hence $2|B|=S G|H|$ and $|H|=1,2,3$. If $|H|=2$, then $|A|=S, 2|C|=2 G^{2}$ and $|B|=S G$. By Lemma 2.1 we get $G \mid A$; it follows that $F(X)=A^{2} X^{2} \pm 2|A| G X-G^{2}=$ $(|A| X \pm G)^{2}-2 G^{2}$, where by Lemma 2.1 we have $2 G^{2} \mid 4(|A| X \pm G)$. If $|H|=1$ or 3, then $4|C|=3 G^{2},|A|=S, 2|B|=S G|H|$. Since $G=1,2$ we must get $G=2, C=-3,|B|=S|H|,|A|=S$. Hence, $F(X)=(S X \pm H)^{2}-H^{2}-3$, where, by Lemma $2.1, H \mid S$. Since $H^{2}+3 \mid 4 H$ when $H=1,3$ we see that $F(X)$ is of Richaud-Degert type.

Now suppose $C=K^{2}$. Since $G^{2} H \mid 4 C$, we get $G H \mid 2 K$ and

$$
\left(\frac{2 B}{S G H}\right)^{2}-\left(\frac{2 K}{G H}\right)^{2}\left(\frac{A}{S}\right)^{2}=\frac{4}{H} ;
$$

thus $|H|=1,2$. Since a difference of two squares can never be 2 , we must have $|H|=1$. Since two squares can differ by 4 only when both are even, we get $2 \mid(2 B /(S G))$ and $2 \mid(2 K / G)$; hence,

$$
|B /(S G)|+(K / G)|A / S|=1
$$

and $B=0,|A|=S, K=G$. Since $F(X)=A^{2} X^{2}+G^{2}$ and $G=1,2$, we get $G^{2} \mid 4 A X$ and $F(X)$ is of Richaud-Degert type.

Note that if $X<0$, we may write $F(X)=A^{2}|X|^{2}-2 B|X|+C$; thus, we may always assume that $X>0$. Also, if we put $X=W+h$, then

$$
F(X)=G(W)=A^{2}(W+h)^{2}+2 B(W+h)+C=A^{\prime 2} W^{2}+2 B^{\prime} W+C^{\prime},
$$

where $A^{\prime}=A, B^{\prime}=A^{2} h+B, C^{\prime}=A^{2} h^{2}+2 B h+C$. We get $\Delta^{\prime}=$ $4 B^{\prime 2}-4 A^{\prime 2} C=4 B^{2}-4 A^{2} C=\Delta,\left(A^{\prime 2}, B^{\prime}\right)=\left(A^{2}, B\right)$, and $\left(A^{\prime 2}, 2 B^{\prime}, C^{\prime}\right)=$ 
$\left(A^{2}, 2 B, C\right)$. Thus, since $B^{\prime}>0$ for $h>-B / A^{2}$, we may assume that $B>0$ for $X$ large enough. Indeed, since

$$
C^{\prime}-G^{4} H^{2}=A^{2}\left(h^{2}-G^{4} H^{2} / A^{2}\right)+B h+C,
$$

we see that $C^{\prime}>G^{4} H^{2}$ when $h>G^{2} H / A \geq 2 G \geq 4$. Thus, we may also assume that $C>G^{4} H^{2}$ for $X$ large enough.

From all of these observations it is clear that if the conditions of Theorem 2.2 hold, and $F(X)$ is not of Richaud-Degert type, then we may assume with no loss of generality that $F(X)=A^{2} X^{2}+2 B X+C$, where $X>0$, $2 \mid A, A>0, C$ is not a perfect integral square, and $B>0, C>G^{4} H^{2}$ for $X$ large enough. To avoid repeating all of these conditions in the sequel, we will simply use the expression $F(W)=A^{2} W^{2}+2 B W+C$ to represent a form satisfying all of these conditions.

3. Continued fractions. Suppose $D$ is a positive integer, not a square, and let $\delta$ be an integer of $\mathbb{Q}(\sqrt{D})$ with trace $t$ and norm $n$. In pursuing the continued fraction expansion of $\delta$ one obtains a sequence $\left(\left(\delta+P_{h}\right) / Q_{h}\right)$ of complete quotients and a sequence $\left(c_{h}\right)$ of partial quotients given by the formulae

$$
\left(\delta+P_{h}\right) / Q_{h}=c_{h}-\left(\bar{\delta}+P_{h+1}\right) / Q_{h} \quad \text { and } \quad \operatorname{Norm}\left(\delta+P_{h+1}\right)=-Q_{h} Q_{h+1} .
$$

Here $\bar{\delta}$ denotes the conjugate of $\delta$, and plainly $t+P_{h}+P_{h+1}=c_{h} Q_{h}$. The usual notation for continued fractions has us write

$$
\delta=\left[c_{0}, c_{1}, \ldots, c_{h},\left(\delta+P_{h+1}\right) / Q_{h+1}\right] .
$$

We denote the convergents $\left[c_{0}, c_{1}, \ldots, c_{h}\right]$ by $x_{h} / y_{h}$. It is often convenient to drop subscripts, writing $x_{h}=x, x_{h-1}=x^{\prime}$, and so forth. Then we have the decomposition

$$
\begin{aligned}
\left(\begin{array}{cc}
x & -n y \\
y & x-t y
\end{array}\right) & =\left(\begin{array}{ll}
x & x^{\prime} \\
y & y^{\prime}
\end{array}\right)\left(\begin{array}{ll}
1 & P \\
0 & Q
\end{array}\right) \\
& =\left(\begin{array}{cc}
c_{0} & 1 \\
1 & 0
\end{array}\right)\left(\begin{array}{cc}
c_{1} & 1 \\
1 & 0
\end{array}\right) \cdots\left(\begin{array}{cc}
c_{h} & 1 \\
1 & 0
\end{array}\right)\left(\begin{array}{ll}
1 & P \\
0 & Q
\end{array}\right)
\end{aligned}
$$

where $P=P_{h+1}, Q=Q_{h+1}$; hence $x^{2}-t x y+n y^{2}=(-1)^{h+1} Q$. In particular, the case $Q=Q_{h+1}=1\left(P_{h+1}=P_{1}=c_{0}\right)$ yields a nontrivial solution

$$
X^{2}-t X Y+n Y^{2}=(X-\delta Y)(X-\bar{\delta} Y)= \pm 1
$$

to "Pell's equation". A central remark is that

Lemma 3.1. A nontrivial solution $X^{2}-t X Y+n Y^{2}= \pm 1$ to "Pell's equation" formally corresponds to a period of $\delta$ in that

$$
\left(\begin{array}{cc}
X & -n Y \\
Y & X-t Y
\end{array}\right)=\left(\begin{array}{cc}
a_{0} & 1 \\
1 & 0
\end{array}\right)\left(\begin{array}{cc}
a_{1} & 1 \\
1 & 0
\end{array}\right) \cdots\left(\begin{array}{cc}
a_{r+1} & 1 \\
1 & 0
\end{array}\right)
$$

entails $\delta=\left[\overline{a_{0}, a_{1}, \ldots, a_{r+1}}\right]$. 
For details see [2] or [3]. Note, however, that the entries in the "period" may not be admissible, as they might not all be positive. For example, $\delta=(\sqrt{D}+1) / 2$ has a periodic expansion of the shape

$$
\left[\overline{a_{0}, a_{1}, \ldots, a_{r}, 0}\right]=\left[a_{0}, \overline{a_{1}, \ldots, a_{r}, 0, a_{0}}\right]=\left[a_{0}, \overline{a_{1}, \ldots, a_{r}+a_{0}}\right] .
$$

The case $Q=1$, signalling a complete period - and thus halfway to two such periods - is a special case of $Q \mid t+2 P$, signalling halfway to a period. We note

Lemma 3.2. Suppose $(\delta+P) / Q$ is a complete quotient of the quadratic integer $\delta$ with norm $n$ and trace $t$. If $Q \mid t+2 P$ then $Q \mid t^{2}-4 n$; and if $Q$ is squarefree and $Q \mid t^{2}-4 n$ then $Q \mid t+2 P$.

Proof. It is easy to verify that every complete quotient has $Q \mid \operatorname{Norm}(\delta+$ $P)$, that is, $Q \mid n+t P+P^{2}$. Hence $4 Q \mid\left(t^{2}-4 n\right)-(t+2 P)^{2}$ and the claims are immediate.

Of course this is well known and says no more than that the $\mathbb{Z}$-module $\langle Q, \delta+P\rangle$ is equal to its conjugate essentially when its norm $Q$ is squarefree and divides the discriminant $t^{2}-4 n$. The point is that it is easy to check that such $\mathbb{Z}$-modules - to wit, with $Q \mid \operatorname{Norm}(\delta+P)$-are $\mathbb{Z}[\delta]$-modules, and thus precisely the ideals of the order $\mathbb{Z}[\delta]$. The condition just mentioned is the ambiguity of the ideal. These matters are discussed in extenso in [2].

We will find it useful to introduce the definitions

$$
L=\left(\begin{array}{ll}
1 & 0 \\
1 & 1
\end{array}\right), \quad R=\left(\begin{array}{ll}
1 & 1 \\
0 & 1
\end{array}\right), \quad J=\left(\begin{array}{ll}
0 & 1 \\
1 & 0
\end{array}\right),
$$

the point being that

$$
\left(\begin{array}{ll}
c & 1 \\
1 & 0
\end{array}\right) J=R^{c}, \quad J\left(\begin{array}{cc}
c & 1 \\
1 & 0
\end{array}\right)=L^{c},
$$

whilst $J^{2}=I$. This notation allows one the alternative of viewing a product

$$
\left(\begin{array}{cc}
c_{0} & 1 \\
1 & 0
\end{array}\right)\left(\begin{array}{cc}
c_{1} & 1 \\
1 & 0
\end{array}\right) \cdots\left(\begin{array}{cc}
c_{h} & 1 \\
1 & 0
\end{array}\right) \cdots
$$

corresponding to a continued fraction expansion, as an $R-L$ sequence

$$
R^{c_{0}} L^{c_{1}} R^{c_{2}} L^{c_{3}} \ldots
$$

Lemma 3.3. If $x^{2}-t x y+n y^{2}= \pm Q$, and $Q \mid t+2 P$, then $x / y$ yields half a period of $\delta$.

Proof. It is convenient to notice that a matrix

$$
\left(\begin{array}{cc}
x & -n y \\
y & x-t y
\end{array}\right) R^{t} J=\left(\begin{array}{cc}
-n y+t x & x \\
x & y
\end{array}\right)
$$


is symmetric. Hence, if $t+2 P=c Q$, we have

$$
\begin{aligned}
\left(\begin{array}{cc}
x & -n y \\
y & x-t y
\end{array}\right)^{2} & =\left(\begin{array}{ll}
x & x^{\prime} \\
y & y^{\prime}
\end{array}\right)\left(\begin{array}{ll}
1 & P \\
0 & Q
\end{array}\right) R^{t} J\left(\begin{array}{cc}
1 & 0 \\
P & Q
\end{array}\right)\left(\begin{array}{cc}
x & y \\
x^{\prime} & y^{\prime}
\end{array}\right) J R^{-t} \\
& =\left(\begin{array}{ll}
x & x^{\prime} \\
y & y^{\prime}
\end{array}\right)\left(\begin{array}{cc}
t+2 P & Q \\
Q & 0
\end{array}\right)\left(\begin{array}{cc}
y & x \\
y^{\prime} & x^{\prime}
\end{array}\right) R^{-t} \\
& =Q\left(\begin{array}{ll}
x & x^{\prime} \\
y & y^{\prime}
\end{array}\right)\left(\begin{array}{ll}
c & 1 \\
1 & 0
\end{array}\right)\left(\begin{array}{cc}
y & x \\
y^{\prime} & x^{\prime}
\end{array}\right) R^{-t},
\end{aligned}
$$

yielding a product of unimodular matrices corresponding to the expansion

$$
\left[c_{0}, c_{1}, \ldots, c_{h}, c, c_{h}, \ldots, c_{1}, c_{0}-t, 0\right]
$$

that is,

$$
\delta=\left[c_{0}, \overline{c_{1}, \ldots, c_{h}, c, c_{h}, \ldots, c_{1}, 2 c_{0}-t}\right] .
$$

Here we use the observation that if the continued fraction $\left[c_{0}, c_{1}, \ldots, c_{h}\right]$ corresponds to the matrix $\left(\begin{array}{ll}x & x^{\prime} \\ y & y^{\prime}\end{array}\right)$, then the matrix $\left(\begin{array}{ll}y & x \\ y^{\prime} & x^{\prime}\end{array}\right)$ corresponds to the expansion $\left[c_{h}, c_{h-1}, \ldots, c_{0}, 0\right]$.

We also point out that if some $Q \mid t+2 P$, then $Q=Q_{n}$ where $n \equiv \operatorname{lp}(\delta) / 2$ $(\bmod \operatorname{lp}(\delta))$ and $P_{n}=P_{n+1}$.

We conclude these "rappels" by recalling that

Lemma 3.4. If $D>0$, and $x, y$ are integers satisfying $x^{2}-D y^{2}=$ $K$ where $|K|<\sqrt{D}$, then $x / y$ is a convergent in the continued fraction expansion of $\sqrt{D}$.

4. A continued fraction expansion of $\sqrt{A^{2} W^{2}+2 B W+C}$. We set out to expand $\sqrt{D}$, where $D=A^{2} W^{2}+2 B W+C$. As in Lemma 2.1, put $S=\operatorname{gcd}(A, B)$; so $S \mid B$. We notice that

$$
\sqrt{D}=A W+B / A-(-\sqrt{D}+A W+B / A),
$$

suggesting we consider the approximation $u / v$ of $\sqrt{D}$, where $v=A / S$ and $u=\left(A^{2} / S\right) W+B / S$. We compute that

$$
u^{2}-D v^{2}=\left(B^{2}-A^{2} C\right) / S^{2}=G^{2} H,
$$

where $H$ is squarefree.

We set $x=B / S, y=A / S$ and remark that

$$
u / v-A W=B / A=\left[c_{0}, c_{1}, \ldots, c_{n}\right]=x / y .
$$

This may appear not well defined. Thus we shall insist that $c_{n} \geq 2$ unless $B / A=0$ or 1 , cases which are excluded by our insistence that $D$ not be of 
Richaud-Degert type. Then, writing $x^{\prime} / y^{\prime}=\left[c_{0}, c_{1}, \ldots, c_{n-1}\right]$, we have

$$
\begin{aligned}
\left(\begin{array}{cc}
u & D v \\
v & u
\end{array}\right) & =R^{A W}\left(\begin{array}{cc}
B / S & A C / S \\
A / S & B / S
\end{array}\right) R^{A W} \\
& =R^{A W}\left(\begin{array}{ll}
x & x^{\prime} \\
y & y^{\prime}
\end{array}\right)\left(\begin{array}{cc}
1 & P \\
0 & G^{2}|H|
\end{array}\right) R^{A W} .
\end{aligned}
$$

Here $\left(x^{\prime} y-y^{\prime} x\right) P=x x^{\prime}-y y^{\prime} C$, which is $\pm P=y^{\prime} A C / S-x^{\prime} B / S$. The square of the matrix above is readily seen $\left({ }^{1}\right)$ to be

$$
\begin{gathered}
R^{A W}\left(\begin{array}{ll}
x & x^{\prime} \\
y & y^{\prime}
\end{array}\right)\left(\begin{array}{cc}
1 & P \\
0 & G^{2}|H|
\end{array}\right) R^{2 A W}\left(\begin{array}{cc}
P & G^{2}|H| \\
1 & 0
\end{array}\right)\left(\begin{array}{cc}
y & x \\
y^{\prime} & x^{\prime}
\end{array}\right) R^{A W} \\
=R^{A W}\left(\begin{array}{ll}
x & x^{\prime} \\
y & y^{\prime}
\end{array}\right)\left(\begin{array}{cc}
2 P+2 A W & G^{2}|H| \\
G^{2}|H| & 0
\end{array}\right)\left(\begin{array}{cc}
y & x \\
y^{\prime} & x^{\prime}
\end{array}\right) R^{A W} .
\end{gathered}
$$

The equation for $P$ and Lemma 2.1 show that always $G H \mid 2 P+2 A W$, so the product has the constant divisor $G H$. In other words, if we "nearly" disregard a possibly unpleasant 2 , we see that

$$
|G H|^{-1}\left(\begin{array}{cc}
u & D v \\
v & u
\end{array}\right)^{2}
$$

is "nearly" a unimodular matrix and "nearly" corresponds to a period of $\sqrt{D}$.

Theorem 4.1. Suppose $G=1$ in Lemma 2.1. Then, if $|H|>1$, we get

$$
\sqrt{D}=\left[A W+c_{0}, \bar{w}, 2(P+A W) /|H|, \overleftarrow{w}, 2\left(A W+c_{0}\right)\right]
$$

where $x / y=B / A=\left[c_{0}, c_{1}, \ldots, c_{n}\right]$. Since we set $\vec{w}=c_{1}, \ldots, c_{n}$ for brevity, we also write $\overleftarrow{w}=c_{n}, c_{n-1}, \ldots, c_{1}$. If $|H|=1$, then

$$
\sqrt{D}=\left[A W+c_{0}, \overline{\vec{w}, 2\left(A W+c_{0}\right)}\right] .
$$

Proof. The claim follows easily from Lemma 3.1 and

$$
\left(\begin{array}{cc}
u & D v \\
v & u
\end{array}\right)^{2}=|H| R^{A W}\left(\begin{array}{cc}
x & x^{\prime} \\
y & y^{\prime}
\end{array}\right)\left(\begin{array}{cc}
2(P+A W) /|H| & 1 \\
1 & 0
\end{array}\right)\left(\begin{array}{cc}
y & x \\
y^{\prime} & x^{\prime}
\end{array}\right) R^{A W} .
$$

Note that if $|H|=1$, then $Q=1, P=P_{1}=c_{0}$.

Theorem 4.2. If $G=2$ in Lemma 2.1 , then $D \equiv 5(\bmod 8)$. If $|H|>1$, then

$$
\frac{1}{2}(\sqrt{D}+1)=\left[\frac{1}{2}\left(A W+c_{0}+1\right), \overline{\vec{w}},(P+A W) /|H|, \overleftarrow{w}, A W+c_{0}\right]
$$

$\left({ }^{1}\right)$ The matrix $\left(\begin{array}{cc}B / S & A C / S \\ A / S & B / S\end{array}\right)$ is false symmetric. Taking its false transpose we get $\left(\begin{array}{cc}G^{2}|H| & P \\ 0 & 1\end{array}\right)\left(\begin{array}{cc}y^{\prime} & x^{\prime} \\ y & x\end{array}\right)=\left(\begin{array}{cc}G^{2}|H| & P \\ 0 & 1\end{array}\right) J \cdot J\left(\begin{array}{cc}y^{\prime} & x^{\prime} \\ y & x\end{array}\right)=\left(\begin{array}{cc}P & G^{2}|H| \\ 1 & 0\end{array}\right)\left(\begin{array}{cc}y & x \\ y^{\prime} & x^{\prime}\end{array}\right)$. 
displays the period of $\frac{1}{2}(\sqrt{D}+1)$. Here, $\left[\frac{1}{2} c_{0}+\frac{1}{2}, \vec{w}\right]=\frac{1}{2} x / y+\frac{1}{2}=\frac{1}{2}(B /(2 A)$ $+1)$. If $|H|=1$, then

$$
\frac{1}{2}(\sqrt{D}+1)=\left[\frac{1}{2}\left(A W+c_{0}+1\right), \overrightarrow{\vec{w}}, A W+c_{0}\right] .
$$

Proof. On referring to Lemma 2.1 we see that if $2 \mid A / S$, then $2 \mid B / S$, which is impossible. If $2 \mid B / S$, then $4 \mid C$ and $4 \mid \operatorname{gcd}\left(A^{2}, 2 B, C\right)$, which is also impossible. Hence, we must have $2 \nmid B / S$ and $2 \nmid A / S$. It follows that $C$ is odd and, since $H \mid C$, that $H$ is odd; hence, $D \equiv 5(\bmod 8)$.

We note that $u \equiv v(\bmod 2)$ and

$$
\left(\begin{array}{cc}
\frac{1}{2}(u+v) & \frac{1}{4}(D-1) v \\
v & \frac{1}{2}(u-v)
\end{array}\right)=\frac{1}{4}\left(\begin{array}{cc}
1 & 0 \\
0 & 2
\end{array}\right) R\left(\begin{array}{cc}
u & D v \\
v & u
\end{array}\right) R^{-1}\left(\begin{array}{cc}
2 & 0 \\
0 & 1
\end{array}\right) .
$$

Also,

$$
\begin{aligned}
\left(\begin{array}{ll}
1 & 0 \\
0 & 2
\end{array}\right) R\left(\begin{array}{ll}
x & x^{\prime} \\
y & y^{\prime}
\end{array}\right)\left(\begin{array}{cc}
1 & P \\
0 & 4|H|
\end{array}\right) R^{-1}\left(\begin{array}{ll}
2 & 0 \\
0 & 1
\end{array}\right) & \\
& =2\left(\begin{array}{cc}
\frac{1}{2}(x+y) & x^{\prime}+y^{\prime} \\
y & 2 y^{\prime}
\end{array}\right)\left(\begin{array}{cc}
2 & P-1 \\
0 & 2|H|
\end{array}\right),
\end{aligned}
$$

whence

$$
\begin{aligned}
\left(\begin{array}{cc}
(u+v) / 2 & \frac{1}{4}(D-1) v \\
v & (u-v) / 2
\end{array}\right)^{2} & \\
= & |H| R^{A W / 2}\left(\begin{array}{cc}
\frac{1}{2}(x+y) & x^{\prime}+y^{\prime} \\
y & 2 y^{\prime}
\end{array}\right) \\
& \times\left(\begin{array}{cc}
(P+A W) /|H| & 1 \\
1 & 0
\end{array}\right)\left(\begin{array}{cc}
y & \frac{1}{2}(x+y) \\
2 y^{\prime} & x^{\prime}+y^{\prime}
\end{array}\right) R^{A W / 2-1} .
\end{aligned}
$$

Our result now follows from Lemma 3.1.

As hinted at earlier, all these expansions are of the shape

$$
\left[b_{0}+\frac{1}{2}(Q W y+s), \overline{b_{1}, \ldots, b_{h}, b+W y, b_{h}, \ldots, b_{1}, 2 b_{0}+Q W y}\right] \text {. }
$$

To sustain this remark we note that above $\left[c_{0}, c_{1}, \ldots, c_{h}\right]=(B / S) /(A / S)$ whence $y=A / S$ and $Q y=Q A / S$. Thus $S=Q$ and $|H|=Q$ throughout. Finally, we observe that all the $\sqrt{A^{2} W^{2}+2 B W+C}$ have the same period length for all $W$ with $W$ large enough to avoid some degenerate cases.

We can also produce the continued fraction expansion of

$$
\sqrt{A^{2} W^{2}+2 B W+C}
$$

in terms of the continued fraction expansion of $\sqrt{C}$. 
Theorem 4.3. If $G=1$ in Lemma 2.1, let $\sqrt{C}=\left[c_{0}, c_{1}, \ldots, c_{n}, \ldots\right]$. Set $\vec{w}=c_{1}, \ldots, c_{n}$, and so $\overleftarrow{w}=c_{n}, \ldots, c_{1}$. Here $Q_{n+1}=|H|$. Then

$$
\sqrt{D}=\left[A W+c_{0}, \overline{\vec{w}}, 2 A W / Q_{n+1}+c_{n+1}, \overleftarrow{w}, 2\left(A W+c_{0}\right)\right]
$$

if $|H|>1$. When $|H|=1$,

$$
\sqrt{D}=\left[A W+c_{0}, \overline{\vec{w}, 2\left(A W+c_{0}\right)}\right] .
$$

Proof. By Lemma 3.4, we know that if

$$
(B / S)^{2}-(A / S)^{2} C=H
$$

is soluble, then $|H|$ must be some $Q_{n+1}$. Further, $n+1=(2 k+1) \pi / 2$, where $\pi=\operatorname{lp}(\sqrt{C}), P=P_{n+1}=P_{n}$, and $c_{n+1}=2 P_{n+1} / Q_{n+1}$. Also, by Lemma 3.1,

$$
\left(\begin{array}{ll}
x & x^{\prime} \\
y & y^{\prime}
\end{array}\right)=\left(\begin{array}{cc}
c_{0} & 1 \\
1 & 0
\end{array}\right) \cdots\left(\begin{array}{cc}
c_{n} & 1 \\
1 & 0
\end{array}\right) .
$$

The result now follows in a similar fashion to that of Theorem 4.1.

We also have a result connecting the continued fraction expansion of $\frac{1}{2}(\sqrt{D}+1)$ to that of $\frac{1}{2}(\sqrt{C}+1)$.

TheOREM 4.4. If we take $G=2$ in Lemma 2.1 and $\frac{1}{2}(\sqrt{C}+1)=$ $\left[c_{0}, c_{1}, \ldots, c_{n}, \ldots\right]$, where $Q_{n+1}=|H|$, then

$$
\frac{1}{2}(\sqrt{D}+1)=\left[A W / 2+c_{0}, \overline{\vec{w}}, A W / Q_{n+1}+c_{n+1}, \overleftarrow{w}, A W+2 c_{0}-1\right]
$$

if $|H|>1$, and when $|H|=1$

$$
\frac{1}{2}(\sqrt{D}+1)=\left[A W / 2+c_{0}, \overline{\vec{w}, A W+2 c_{0}-1}\right] .
$$

Proof. As in the proof of Theorem 4.3 we have $n+1=(2 h+1) \pi / 2-$ recall that $\pi=\operatorname{lp}\left(\frac{1}{2}(\sqrt{C}+1)\right)-Q_{n+1}=|H|, P_{n+1}=P_{n+2}, Q_{n+1} \mid 2 P_{n+1}+$ $1, P=2 P_{n+1}+1$, and $c_{n+1}=P / Q_{n+1}$. Since by Lemma 3.1 we have

$$
\left(\begin{array}{cc}
(x+y) / 2 & x^{\prime}+y^{\prime} \\
y & 2 y^{\prime}
\end{array}\right)=\left(\begin{array}{cc}
c_{0} & 1 \\
1 & 0
\end{array}\right)\left(\begin{array}{cc}
c_{1} & 1 \\
1 & 0
\end{array}\right) \cdots\left(\begin{array}{cc}
c_{n} & 1 \\
1 & 0
\end{array}\right),
$$

we get our result as in the proof of Theorem 4.2.

Notice that in the case of Theorem 4.3 we get

$$
\operatorname{lp}(\sqrt{D})= \begin{cases}(2 k+1) \pi & \text { if }|H|>1, \\ (2 k+1) \pi / 2 & \text { if }|H|=1,\end{cases}
$$

where $\pi=\operatorname{lp}(\sqrt{C})$; hence $\operatorname{lp}(\sqrt{D})$ can be as large as we want by selecting $k$ large enough. There is, of course, a similar result in the case of Theorem 4.4.

5. The final case. Our task at this point is still incomplete. We undertook to produce the continued fraction expansion of $\sqrt{D}$, but we have only 
that of $(\sqrt{D}+1) / 2$ in the case of $G=2$. However, we can get a result, like that of Theorem 4.3, in which we relate the continued fraction expansion of $\sqrt{D}$ to that of $\sqrt{C}$. First we need some results concerning the continued fraction expansion of $\sqrt{C}$.

Theorem 5.1. Let $C \equiv 5(\bmod 8)$ and suppose that in the continued fraction expansion of $\sqrt{C}$ we get $4 \mid Q_{m+1}, 1<\frac{1}{4} Q_{m+1}<C, \frac{1}{4} Q_{m+1}$ squarefree, and $\frac{1}{4} Q_{m+1} \mid C$. If $m$ is the least nonnegative integer for which these conditions hold, then the fundamental unit $\varepsilon$ of the order $\mathcal{O}=\langle 1, \sqrt{C}\rangle$ is given by

$$
\varepsilon=\delta^{6} / Q_{m+1}^{3},
$$

where $\delta=x_{m}+\sqrt{C} y_{m}$.

Proof. We know that $2 Q_{m+1} \mid x_{m}\left(x_{m}^{2}+3 C y_{m}^{2}\right)$ and $2 Q_{m+1} \mid y_{m}\left(C y_{m}^{2}+\right.$ $\left.3 x_{m}^{2}\right)$ by the same reasoning as that used above. Hence $\nu=\lambda^{3} /\left(2 Q_{m+1}\right)$ $\in \mathcal{O}$. Now $|n(\nu)|=\frac{1}{4} Q_{m+1}$ and $\frac{1}{4} Q_{m+1} \mid C$. Thus, $\varepsilon \equiv 4 \nu^{2} / Q_{m+1} \in \mathcal{O}$ and $n(\varepsilon)=1$. It follows that $\varepsilon=\lambda^{6} / Q_{m+1}^{3}$ is a unit of $\mathcal{O}$. Also there must exist some $\theta=x_{r}+y_{r} \sqrt{C}$ such that $Q_{r+1}=Q_{m+1}$ and $\eta=4 \theta^{2} / Q_{m+1}$ is the fundamental unit of $\mathcal{O}$. By definition of $\lambda$ we have $\lambda<\theta$; consequently, $\lambda^{2}<\eta$ and $\varepsilon<\eta^{3}$. It follows that $\varepsilon=1, \eta$, or $\eta^{2}$. If $\varepsilon=1$, we get $\lambda^{2} / Q_{m+1}=1$ and $\lambda^{2}=Q_{m+1}$. If $\varepsilon=\eta^{2}$, we get $\left(\lambda^{3} /\left(Q_{m+1} \eta\right)\right)^{2}=Q_{m+1}$. In either case we find that $Q_{m+1}=\alpha^{2}$ where $\alpha \in \mathcal{O}$. If $\alpha=a+b \sqrt{C}$, then $a b=0$. If $b=0$, then $Q_{m+1}=a^{2}$; if $Q=0$, then $\frac{1}{4} Q_{m+1}=(b / 2)^{2} C$. Thus $\varepsilon=\eta$.

Corollary 5.2. If $\mu=2 \lambda^{2} / Q_{m+1}$, then $\mu=x_{n}+\sqrt{C} y_{n}$, where $n$ is the least nonnegative integer such that $Q_{n+1}=4$. Also, $\nu=x_{p}+\sqrt{C} y_{p}$, where $p=\pi / 2$ and $\pi=\operatorname{lp}(\sqrt{C})$.

COROLlary 5.3. If $Q_{k+1}=Q_{m+1}$ and $k<\pi$, then $k=m$ or $k=$ $\pi-m-2$; if $Q_{k+1}=4$ and $k<\pi$, then $k=n$ or $k=\pi-n-2$; if $Q_{k+1}=Q_{p+1}$ and $k<\pi$, then $k+1=\pi / 2$.

COROllary 5.4. Suppose $r=m+k \pi$ (for some $k \geq 0$ ). Set $2\left(x_{r}+\sqrt{D} y_{r}\right)^{2} / Q_{m+1}=x_{s}+\sqrt{C} y_{s}, \quad\left(x_{r}+\sqrt{C} y_{r}\right)^{3} /\left(2 Q_{m+1}\right)=x_{t}+\sqrt{C} y_{t}$. Then $s=n+2 k \pi$ and $t=p+3 k \pi$. If $r=-m+(k+1) \pi(k \geq 0)$, then $s=-n+2(k+1) \pi$ and $t=-p+3(k+1) \pi$.

Corollary 5.5. Let $r, s$, and $t$ be defined as in Corollary 5.4. We must have $s>r+1$ and, unless $y_{r}=1$, we must have $t>s+1$.

Proof. We have $\left|x_{k}-\sqrt{C} y_{r}\right|<1$ as a property of the convergents in a continued fraction expansion; hence, $x_{m}+\sqrt{C} y_{m}>Q_{m+1}$, and therefore $\mu>\nu>\lambda$. It follows that $p>n>m$. Thus, we must have $s>r$. If $s=r+1$, then $4 \mid Q_{r+1}$ and $4 \mid Q_{r+2}$, which means that $P_{r+2}^{2}-C \equiv 0(\bmod 16)$, which 
is impossible as $C \equiv 5(\bmod 8)$. If $t=s+1$, then we can only have $r=m$, $s=n$, and $t=p$. Now

$$
\begin{aligned}
\left(\begin{array}{cc}
x_{m} & C y_{m} \\
y_{m} & x_{m}
\end{array}\right) & =Q_{n+1}\left(\begin{array}{cc}
x_{n} & C y_{n} \\
y_{n} & x_{n}
\end{array}\right)^{-1}\left(\begin{array}{cc}
x_{p} & C y_{p} \\
y_{p} & x_{p}
\end{array}\right) \\
& =\left(\begin{array}{cc}
Q_{n+1} & -P_{n+1} \\
0 & 1
\end{array}\right)\left(\begin{array}{cc}
c_{n+1} & 1 \\
1 & 0
\end{array}\right)\left(\begin{array}{ll}
1 & P_{p+1} \\
0 & Q_{p+1}
\end{array}\right),
\end{aligned}
$$

by our observations at the beginning of Section 3. Hence, $y_{r}=y_{m}=1$. We note that if $t=s+1$, then $v=A / S=y_{r}=1$ and $u^{2}-D=4 Q_{r+1}$. Since $u=\left(A^{2} / S\right) W+B / S$ and $Q_{r+1}=|H|$ and $H$ is odd, we get $4 Q_{r+1} \mid 4 u$ and $D$ is of Richaud-Degert type.

In the following theorem we will, as usual, assume that $D$ is not of Richaud-Degert type and that therefore $t>s+1$. We are now able to derive the form of the continued fraction expansion of $\sqrt{D}$.

Theorem 5.6. If $G=2$ in Lemma 2.1, then for $\sqrt{C}=\left[c_{0}, c_{1}, \ldots, c_{k}, \ldots\right]$, we must get $G^{2}|H|=Q_{r+1}$ for $r=m+k \pi$, or $r=-m+(k+1) \pi$ $(k \geq 0)$, where $m$ is defined in Theorem 5.1 and $\pi$ is defined in Corollary 5.2. Put $\overrightarrow{w_{1}}=c_{1}, \ldots, c_{p} ; \overrightarrow{w_{2}}=c_{r+2}, \ldots, c_{s} ; \overrightarrow{w_{3}}=c_{s+2}, \ldots, c_{t}$, where $s$ and $t$ are given by Corollary 5.4. The continued fraction expansion of $\sqrt{D}$ is given by

$$
\begin{aligned}
& {\left[A W+c_{0}, \overrightarrow{w_{1}}, 2 A W / Q_{m+1}+c_{m+1}, \overrightarrow{w_{2}}, \frac{1}{2} A W+c_{n+1}, \overrightarrow{w_{3}}, 2 A W / Q_{p+1}+c_{p+1},\right.} \\
& \left.\overleftarrow{w_{3}}, \frac{1}{2} A W+c_{n+1}, \overleftarrow{w_{2}}, 2 A W / Q_{m+1}+c_{m+1}, \overleftarrow{w_{1}}, 2 A W+2 c_{0}\right]
\end{aligned}
$$

when $4|H|=Q_{m+1} \neq 4$. If $4|H|=Q_{m+1}=4$, it is given by

$$
\sqrt{D}=\left[A W+c_{0}, \overrightarrow{\overrightarrow{w_{1}}}, \frac{1}{2} A W+c_{m+1}, \overrightarrow{w_{2}}, \frac{1}{2} A W+c_{n+1}, \overrightarrow{w_{3}}, 2 A W+2 c_{0}\right] .
$$

P r o o f. The first part of the theorem follows from Lemma 3.4 and Corollary 5.4. We note that

$$
\begin{aligned}
\left(\begin{array}{cc}
x_{s} & C y_{s} \\
y_{s} & x_{s}
\end{array}\right) & =\frac{2}{Q_{m+1}}\left(\begin{array}{cc}
x_{r} & C y_{r} \\
y_{r} & x_{r}
\end{array}\right)^{2}, \\
\left(\begin{array}{cc}
x_{t} & C y_{t} \\
y_{t} & x_{t}
\end{array}\right) & =\frac{1}{2}\left(\begin{array}{cc}
x_{s} & C y_{s} \\
y_{s} & x_{s}
\end{array}\right)\left(\begin{array}{cc}
x_{r} & C y_{r} \\
y_{r} & x_{r}
\end{array}\right),
\end{aligned}
$$

and

$$
\left(\begin{array}{cc}
1 & P_{k+1} \\
0 & Q_{k+1}
\end{array}\right) R^{2 A W}\left(\begin{array}{cc}
0 & Q_{k+1} \\
1 & -P_{k+2}
\end{array}\right)^{-1}=\left(\begin{array}{cc}
2 A W / Q_{k+1}+c_{k+1} & 1 \\
1 & 0
\end{array}\right) .
$$


For $i+1<j$, define

$$
\begin{aligned}
T_{i, j} & =\left(\begin{array}{ll}
x_{i+1} & x_{i} \\
y_{i+1} & y_{i}
\end{array}\right)^{-1}\left(\begin{array}{ll}
x_{j} & x_{j-1} \\
y_{j} & y_{j-1}
\end{array}\right) \\
& =\left(\begin{array}{cc}
c_{i+2} & 1 \\
1 & 0
\end{array}\right)\left(\begin{array}{cc}
c_{i+3} & 1 \\
1 & 0
\end{array}\right) \cdots\left(\begin{array}{cc}
c_{j} & 1 \\
1 & 0
\end{array}\right) .
\end{aligned}
$$

Then we have

$$
T_{i, j}\left(\begin{array}{cc}
1 & P_{j+1} \\
0 & Q_{j+1}
\end{array}\right)=\left(\begin{array}{cc}
0 & Q_{i+1} \\
1 & -P_{i+2}
\end{array}\right)\left(\begin{array}{cc}
x_{i} & C y_{i} \\
y_{i} & x_{i}
\end{array}\right)^{-1}\left(\begin{array}{cc}
x_{j} & C y_{j} \\
y_{j} & x_{j}
\end{array}\right) .
$$

Thus,

$$
\begin{aligned}
\left(\begin{array}{cc}
u & D v \\
v & u
\end{array}\right)^{3}= & R^{A W}\left(\begin{array}{ll}
x_{r} & x_{r-1} \\
y_{r} & y_{r-1}
\end{array}\right)\left(\begin{array}{ll}
1 & P_{m+1} \\
0 & Q_{m+1}
\end{array}\right) \\
& \times R^{2 A W}\left(\begin{array}{cc}
x_{r} & C y_{r} \\
y_{r} & x_{r}
\end{array}\right) R^{2 A W}\left(\begin{array}{cc}
x_{r} & C y_{r} \\
y_{r} & y_{r}
\end{array}\right) R^{A W} \\
= & 2 Q_{m+1} R^{A W}\left(\begin{array}{cc}
c_{0} & 1 \\
1 & 0
\end{array}\right) T_{-1, r}\left(\begin{array}{cc}
2 A W / Q_{m+1}+c_{m+1} & 1 \\
1 & 0
\end{array}\right) \\
& \times T_{r, s}\left(\begin{array}{cc}
A W / 2+c_{n+1} & 1 \\
1 & 0
\end{array}\right) T_{s, t}\left(\begin{array}{cc}
1 & P_{p+1} \\
0 & Q_{p+1}
\end{array}\right) R^{A W}
\end{aligned}
$$

We set

$$
\begin{aligned}
K= & \left(\begin{array}{cc}
c_{0} & 1 \\
1 & 0
\end{array}\right) T_{-1, r}\left(\begin{array}{cc}
2 A W / Q_{m+1}+c_{m+1} & 1 \\
1 & 0
\end{array}\right) T_{r, s} \\
& \times\left(\begin{array}{cc}
A W / 2+c_{n+1} & 1 \\
1 & 0
\end{array}\right) T_{s, t},
\end{aligned}
$$

and note that $K\left(\begin{array}{ll}1 & P_{p+1} \\ 0 & Q_{p+1}\end{array}\right)$ is false symmetric. Hence,

$$
\frac{1}{Q_{m+1}^{3}}\left(\begin{array}{cc}
u & D v \\
v & u
\end{array}\right)^{6}=K\left(\begin{array}{cc}
2 A W / Q_{p+1}+c_{p+1} & 1 \\
1 & 0
\end{array}\right) K^{*} R^{A W},
$$

where $K^{*}=K^{t} J$, with $K^{t}$ the transpose of $K$ and, as above, $J=\left(\begin{array}{ll}0 & 1 \\ 1 & 0\end{array}\right)$. Then our claimed continued fraction expansion follows immediately from Lemma 3.1 and our observations at the beginning of this section. Further, if $|H|=1$, then $Q_{m+1}=Q_{n+1}=4, c_{p+1}=2 c_{0}$, and we have the palindrome

$$
\overrightarrow{w_{1}}, c_{m+1}, \overrightarrow{w_{2}}, c_{n+1}, \overrightarrow{w_{3}}=\overleftarrow{w_{3}}, c_{n+1}, \overleftarrow{w_{2}}, c_{m+1}, \overleftarrow{w_{1}}
$$

This information yields the expansion of $\sqrt{D}$ claimed for the case $|H|=1$.

Thus, if $G=2$, we get, for some $k \geq 0$,

$$
\operatorname{lp}(\sqrt{D})= \begin{cases}(6 k+1) \operatorname{lp}(\sqrt{C}) & \text { if }|H|>1 \\ (6 k+1) \operatorname{lp}(\sqrt{C}) / 2 & \text { if }|H|=1\end{cases}
$$




\section{References}

[1] R. A. Mollin, Quadratics, CRC Press, Boca Raton, 1996.

[2] R. A. Mollin and A. J. van der Poorten, A note on symmetry and ambiguity, Bull. Austral. Math. Soc. 51 (1995), 215-233.

[3] R. A. Mollin, A. J. van der Poorten and H. C. Williams, Halfway to a solution of $X^{2}-D Y^{2}=-3$, J. Théor. Nombres Bordeaux 6 (1994), 421-459.

[4] A. Schinzel, On some problems of the arithmetical theory of continued fractions, Acta Arith. 6 (1961), 393-413.

[5] - On some problems of the arithmetical theory of continued fractions II, ibid. 7 (1962), 287-298. Corrigendum, ibid. 47 (1986), 295.

[6] H.-J. Stender, Über die Grundeinheit der reell-quadratischen Zahlkörper $\mathbb{Q}\left(\sqrt{A^{2} N^{2}+B N+C}\right)$, J. Reine Angew. Math. 311/312 (1979), 302-306.

[7] M. A. Stern, Zur Theorie der periodischen Kettenbrüche, ibid. 8 (1857), 1-102.

School of MPCE

Macquarie University

Sydney, New South Wales 2109

Australia

E-mail: alf@mpce.mq.edu.au
Department of Computer Science University of Manitoba Winnipeg, Manitoba Canada R3T 2N2

E-mail: williams@cs.umanitoba.ca 